\title{
The Mathematics in the Social Studies Textbook: A Critical Content Analysis and Implications for Students' Reasoning
}

\author{
Flávio S. Azevedo, Michele J. Mann \\ STEM Education, Department of Curriculum and Instruction, The University of Texas at Austin, Austin, TX, USA \\ Email: flavio@austin.utexas.edu,mjmann@utexas.edu
}

How to cite this paper: Azevedo, F. S., \& Mann, M. J. (2019). The Mathematics in the Social Studies Textbook: A Critical Content Analysis and Implications for Students' Reasoning. Creative Education, 10, 1-25. https://doi.org/10.4236/ce.2019.101001

Received: December 21, 2018

Accepted: January 6, 2019

Published: January 9, 2019

Copyright (c) 2019 by authors and Scientific Research Publishing Inc. This work is licensed under the Creative Commons Attribution International License (CC BY 4.0).

http://creativecommons.org/licenses/by/4.0/

\section{c) (i) Open Access}

\begin{abstract}
We report on a case study of the mathematical content of a $10^{\text {th }}$ grade social studies textbook. We develop our case in three analytical steps. First, we identify, describe, and categorize the full range of mathematics in the book. Put simply, we ask: What mathematical forms (e.g., Cartesian graphs and problems) do we find and what kinds of mathematical work do they require? Second, we characterize and critically evaluate the mathematics content in the textbook, focusing in particular on the kinds of mathematics literacy and student reasoning that the book fosters. Third and finally, we operationalize a measure of the "density" of mathematics in the textbook-that is, an estimate of the presence and pervasiveness of mathematical objects and practices relative to other disciplinary contents and track how such "density" has changed over the past three editions of the same volume. Doing so helps us further contextualize and elaborate the prior analyses, but also surfaces shifts in the patterns of mathematics presence in that textbook series, including the growing encroachment of mathematics exercising and visual/representational presence.
\end{abstract}

\section{Keywords}

Social Studies Education, Mathematics, Student Reasoning, Textbook Analysis, $10^{\text {th }}$ Grade (USA)

\section{Introduction}

The centrality of textbooks to organizing all aspects of school life has long been recognized (Apple \& Christian-Smith, 1991). In the classroom, in particular, textbooks are traditionally the core mediator of learning activities and instructional delivery (Apple, 2007). Much beyond K-12 classroom walls, however, the 
importance and "weight" of textbooks is felt in arguments and discussions regarding educational policy, curriculum development, and teacher education.

For these reasons, the systematic study of textbook contents has had a long career in educational research (e.g., Lee, 2010; Marino, 2011). Among these, social studies textbooks have seen a fair share of attention (Nichols, 2003; Roberts, 2014b; Ward, 2006). And yet, to date, no research has investigated the mathematical content of social studies textbooks. This seems to be a glaring omission, given the presumed importance of mathematics for the various disciplines within the purview of social studies (e.g., history, geography, and political economy), and indeed for the curriculum as a whole.

In this paper, therefore, we pursue the project of systematically documenting the mathematical content of a social studies textbook and critically evaluating its forms, particularly as it regards the kinds of student reasoning that such content affords. We do so through an in-depth case study of a single $10^{\text {th }}$ grade (USA) social studies textbook and its prior two editions. Because the study is the first of its kind, our choice of a case study is meant to reveal the breadth, depth, and general character of the mathematics presence in the book-a foundation upon which others can improve and generalize.

We develop our case in three steps. First, we identify, describe, and categorize the full range of mathematics manifested in the book. Specifically, we ask: What mathematical forms (say, representations and problems/tasks) do we find? How is each mathematical form manifested? For example, what kinds of mathematics problems and tasks are there? What mathematical disciplinary content do they cover? In addressing these questions, we advance a descriptive account aimed to convey a rich picture of the mathematics in the social studies textbook and to lay the groundwork for the subsequent analytical steps.

In the second step, we characterize and critically evaluate the mathematics content in the textbook, focusing in particular on the kinds of mathematics literacy and thinking practices that the book fosters (Gutstein, 2007; Stevens, Wineburg, Herrenkohl, \& Bell, 2005). We ask: What is the nature of the mathematical reasoning engendered by such problems/tasks? What problem solving practices are instantiated in the book? How are mathematical forms used to support students' developing thinking in history, geography, and political economy? Crucially, how do they compare and contrast to other approaches to mathematical problem solving in the social studies curriculum (e.g., Hollister, 1995)?

Third and finally, we operationalize a measure of the "density" of mathematics in the textbook-that is, an estimate of the presence and pervasiveness of mathematical objects and practices, relative to other disciplinary contents and track how such "density" has changed over the past three editions of the same volume. Doing so helps us further contextualize and elaborate the analyses on prior steps and, importantly, its surfaces shifts in the patterns of mathematics presence in that textbook series, including a growing encroachment of mathematics exercising and visual presence. 


\section{The Textbook and Its Structure}

The subject of our study is a textbook titled The Americans: United States History Since 1877 (Texas Edition) and it was published in 2015. The book is one of several texts approved for adoption by the Texas Education Agency and it supports Common Core Standards.

The volume has 923 pages, in addition to standard reference, introductory, and various "special" sections. The substantive content is organized into seven units, each covering a distinct era in U.S. history. Units themselves are divided into chapters-27 in all-that cover chronologically thematic, and thus sometimes overlapping historical periods.

Crucial to our goals here, each individual page has a complex graphical layout. Such layout choice seems to follow from a premise stated in the book's introductory remarks: “...students today need to read in ways that earlier generations did not... and must deal with information that come in various formats" (p. T11), including multiple columns of text as well as graphics, photographs, maps, political cartoons, and many other pictorial forms. Apparently in an effort to achieve the effect of multi-modal, non-linear reading, the textbook designers segmented each page into distinct, visually well-bounded areas around which the main narrative text "snakes". Figure 1 reproduces one such page.

In the figure, we can identify four separate textual/pictorial areas, three of which are distinctly labelled Personal Voice, Historical Spotlight, and Skillbuilder Interpreting Graphs; the fourth, unlabelled part of the page is the main narrative text. We name call-out box (or simply call-out) those distinct page layout areas (or structures) that are not the main narrative text.

Call-out boxes are differentially integrated with the main text and, as a group, call-outs usually occupy most of the real estate of any single page. Referring back to Figure 1, on the top right of the page we find a Historical Spotlight call-out box. A Historical Spotlight always presents a short textual elaboration of particular historical issues in the current narrative context-in the current case, a note on Vice President Johnson's strong influence on the development of the American space program.

In the middle of the page, a Personal Voice call-out reproduces an excerpt of President Kennedy's September 1962 address to the nation in which he alluded to the challenge and promise of the space program. Personal Voice call-outs display a first-person account of a historical event or situation by a historical actor or directly reproduces such figure's statements/writing.

Finally, at the bottom of the page, there is a Skillbuilder Interpreting Graph (“U.S. Space Race Expenditures, 1959-1975”), which we analyse in detail later. For now, note the pictorial representations (of multiple types, in this case) and proposed two-question problem format typical of this kind of call-out box.

There are more types of call-outs-e.g., Economic Background, World Stage, Science \& Technology, and Geography Skillbuilder-always associated with specific disciplinary content, as well as various "special" sections within chapters; 


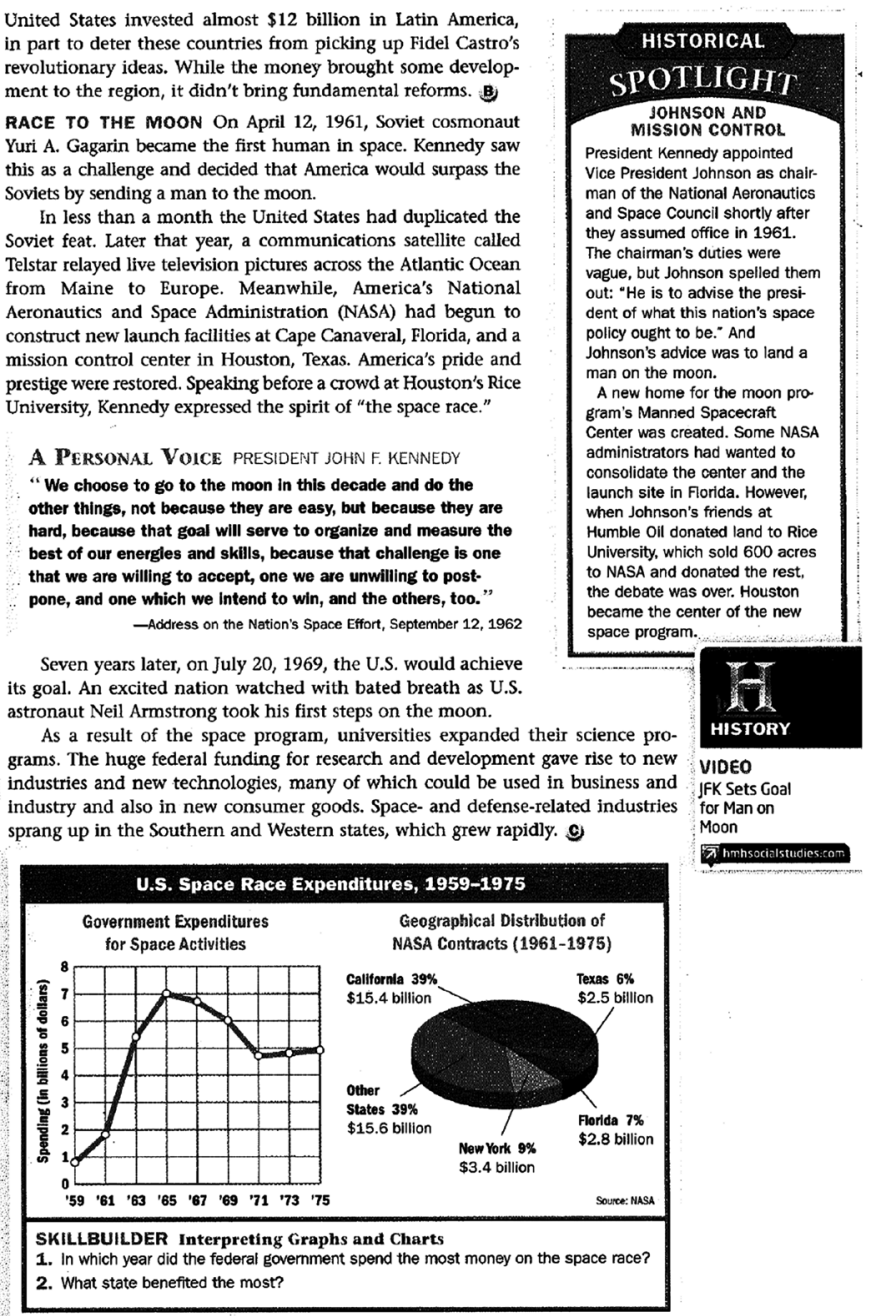

Figure 1. A page from The Americans: United State History Since 1877.

any such structure may or may not contain mathematics material. Other book structures emerged as analytically consequential, as we will see.

\section{Conceptual Framework}

Given our goals, we were faced with the challenge of crafting a framework of study for an as-yet unstudied problem - that is, describing, categorizing, "quantifying," and critically appraising the mathematics content in a textbook of an entirely different disciplinary realm. We thus found it necessary to borrow from various theoretical perspectives to textbook analysis (Apple \& Christian-Smith, 1991; Nichols, 2003; Roberts, 2014b), including both generic and content-specific frames of textbook (Nichols, 2003) and textual (Janks, 2010) analysis, all of which we blend and extend in light of the conceptual specificities of our research context.

As point of departure, we take it that the nature of the mathematics in the book is a function of both the particular material forms (e.g., representations, practice problems or open-ended problems) in which mathematics is expressed 
and the book structures (e.g., a Skillbuilder call-out or end-of-chapter tutorial section) that frame and present such content to readers. Put differently, the meaning and relevance of various textual elements take shape relationally, within the context of the page and the book as a whole (Janks, 2010), and this is true of mathematical objects (broadly conceived) as well. This approach seeks to do justice to the fundamental organizational units/structures of the book, as well as the distinct nature of the various disciplinary contents crisscrossing throughout the volume.

Beginning with mathematics content, then, our initial conceptual problem is to define what exactly qualifies as mathematics within the context of the book. We tackle this issue by observing, first, that STEM disciplines-mathematics perhaps paradigmatically-are founded on myriad representational forms (or inscriptions) that professionals constantly adapt, modify, or create anew, and which are central to professional STEM discourse and practice (Latour \& Woolgar, 1986; Pickering, 1995). Common representational forms include equations and various symbolic systems, numerical tables, Cartesian graphs, diagrams, models, drawings, and many others (Coopmans et al., 2014). In mathematics classrooms as well, teaching and learning are centrally articulated around the representational nature of the discipline (Greeno \& Hall, 1997). Our first focal concern, therefore, is the overall representational/inscriptional landscape through which mathematics concepts and skills are instantiated in the book.

Second, it has long been noted that problem solving is at the core of professional mathematics practice (Schoenfeld, 1992). Indeed, at a very general level, the work of mathematicians has often been described as the work of solving problems (Pólya, 1945). While there might be no consensus definition of mathematical problem solving, problems of some kind are often the single organizing principle driving students' classroom work and teachers' orchestrating of such activities. When characterizing the mathematics content in The Americans, therefore, we also focus on material and pedagogical forms that are broadly task-like in that they afford some active doing of mathematics. Questions and problems are exemplars of such forms, but many other forms exist as well (e.g., lessons or tutorials on mathematical skills). Put simply, for our purposes, what counts as mathematics is the doing of it, rather than the reading of it (e.g., as embodied in the main narrative text).

To delve critically into the nature of mathematical problems in the book, we place such problems within a landscape of frames for understanding mathematical problem solving and its instructional uses. To do so, we adapt from Schoenfeld's (2007) observations on the many roles that mathematics problems and problem solving have historically served in classrooms (see also Stanic \& Kilpatrick, 1988). For example, very often problems function as means to focused instructional ends, such as a "justification for teaching math." This is generally done by attempting to link the problem to real-world contexts and use, thus implicitly or explicitly implying the usefulness of mathematics. Problems may also function as means to "motivating new subject topics" in the curricu- 
lum, on the premise that once one has learned the material one will be able to solve such problems. Also, problems commonly serve as a context for "routine exercising" of a procedure/method, which has typically been demonstrated by the teacher or textbook. As we will expand upon later, there are additional roles that problems and problem solving take on whenever mathematics instruction is enacted; frequently, they will serve many such roles simultaneously. Juxtaposing these roles when looking at the various types of mathematics problems populating The Americans will lend us a tool to characterize them.

Finally, in light of the above, we operationalize a measure of the "density" of mathematics content first by drawing from our focus on representational/visual aspects of such content and some well-known, general methods of textbook analysis (Nichols, 2003). Specifically, "real-estate" usage and/or frequency of occurrence of any textual element is indicative of its influence on the volume and its overall discourse (Janks, 2010; Nichols, 2003; Roberts, 2014a, 2014b; Wade, 1993). For instance, studying the frequency of occurrence of particular words is a common method to show narrative biases in textbooks (Sleeter \& Grant, 1991). Following these approaches, our measure of mathematical "density" will draw directly from counts of the sheer material presence of mathematical objects in the textbook. ${ }^{1}$

Beyond its material, spatial presence, moreover, we will argue that the "density" of mathematics also includes the activities that the content might engender and the multiple contexts of mathematics use the book presents as a whole. As a measure of influence of a textual element (math, in this case), "density" is therefore also fundamentally related to the work it requires from students.

\section{Method}

Our methods follow straightforwardly from our research questions and the sequential nature of the research steps. We began by scrutinizing the book page by page and recording any instance of mathematics, its general form, content, and placement within the book. We also mapped out all call-out types and various other book structures and respective disciplinary content, including science and technology and social sciences-centred ones (e.g., history and geography). These provided the basis for comparisons regarding the relative "density" of mathematics content.

With all mathematics objects surveyed, we then carried out the descriptive work that grounded all subsequent analysis. To aid this process, we photocopied all pages containing mathematics objects so that they could be juxtaposed and compared along several dimensions. Continuously elaborating relationships between various objects led to refinements in descriptive detail and accuracy. Throughout the analyses, we show samples of different mathematical forms as

${ }^{1}$ Recall that, following contemporary trends in print and electronic media (Janks, 2010), the overall content of The Americans is heavily pictorial, irrespective of disciplinary content. Focusing on pictorial/representational forms of mathematics respects the defining features of the book and makes possible comparisons across disciplinary contents. 
means of illustrating the study's raw data and their treatment.

We then categorized these materials based on emergent themes in our analysis. For example, we sorted representational forms, call-outs, and "special" sections into different categories and further elaborated them into subcategories. We coded and counted these objects and began to establish some baseline measures of "density".

Similarly, in the case of mathematical questions/tasks, we coded each instance according to the kind of mathematical work required for its solution. We then compared and contrasted them to other models of mathematical problem solving in the social studies, focusing particularly on the mathematics thinking practices that each engenders.

Finally, we ran the same analyses on two prior editions of the same volume.

\section{Analysis (1): Describing and Categorizing the Mathematics in the Textbook}

We begin with the foundational work of describing the full variety of material forms that mathematics assumes in the textbook, as well as categorizing and base-counting such content. Because we are interested in the mathematics content and its relation to how it is presented in the book, we organize our descriptive analysis around the various types of book structures in which mathematics appears. This approach allows us to simultaneously consider the general organization of the book and the mathematics that it houses.

\subsection{Skillbuilder Interpreting Graphs}

This call-out structure is where mathematics appears most systematically throughout the book. There are 31 such Skillbuilders, or slightly more than one per chapter, on average. All have the same general format-namely, one or more representations that display some socially relevant data, followed by one to three questions (almost always two) (Figure 1). In total, 48 mathematical representations appear across these Skillbuilders: 28 line graphs, nine pie charts, five bar graphs, four numerical tables, and two infographics.

Overall, 61 questions are posed across Skillbuilder Interpreting Graphs call-outs; 45 (or 73.7\%) of those are math-focused in that the question involves exclusively mathematics work and asks nothing of historical/geographical thinking. The remaining (16, or $26.3 \%)$ questions integrate such mathematics into reasoning about a social studies issue.

As we coded the math-focused questions according to the mathematical work they entailed, six categories of questions emerged. Thirteen questions (or 28.9\%) required "computing" values (e.g., subtracting values in a numerical table or fetching values from a bar graph, then adding them up), 10 questions (22.2\%) required "identifying trends" in a given representation (e.g., growth or decline in a graph), eight questions (17.8\%) required one to "assess slope" in a line graph, seven questions (15.6\%) asked one to "find $\mathrm{min} / \mathrm{max}$ " values in a representation (e.g., the highest/lowest points in a bar or line graph), four questions (8.9\%) re- 
quired computing "\% change" (e.g., the percent growth/decline in per capita spending over a period in a line graph), and finally three questions (6.7\%) required one to "read values" from a mathematical representation (e.g., to fetch a given $x / y$ coordinate in a graph).

To exemplify such questions, let us return to Figure 1. The Skillbuilder in question is labelled "U.S. Space Race Expenditures, 1959-1975" and it presents two distinct mathematical representations-a line graph depicting "Government Expenditures for Space Activities" (1959-1975) and a pie chart showing the percentage of total expenditures allocated to individual states by the space program in that period. The questions read: "1) In which year did the federal government spend the most money on the space race? 2) What state benefitted the most?" (p. 681). Both questions were coded "find $\mathrm{min} / \max$ " values because their solution requires scanning the pictorial representations provided in search of their single, maximum value. We will argue that both questions are of modest instructional value, mathematically and historically.

\subsection{Geography Skillbuilder}

Similar to Skillbuilder Interpreting Graphs, a Geography Skillbuilder presents one or more representations-a map of some sort (say, geographical or political), overlaid with other representations and/or information-and one to three questions (typically two) probing those content. In the case of Geography Skillbuilders containing some form of mathematics, such "overlays" might be a numerical table illustrating voting results or numerical annotations showing changing land areas. In all, there are 55 Geography Skillbuilders, 13 (or 23.6\%) of which have either/both a mathematical representation or/and problem; three such Skillbuilders are math-dominated. In all, four mathematical representations show in these call-outs: one pie chart, one set of bar graphs, and two numerical tables.

Fourteen (14) math-based questions appeared across these 13 Skillbuilders. Many such questions required simple forms of math, such as "counting" entities fitting a criterion (e.g., number of states receiving aid during the spatial program efforts of 1950s and 1960s), which appeared four times, and "find min/max" values (say, on a map overlaid with numbers), which appeared three times. More computationally involved problems include "estimate (distance/area)" and "compute (with map scale)", which appeared three times, and "rate of progress", which appeared twice.

To illustrate, a Geography Skillbuilder labelled "Shrinking Native American Land, and Battle Sites" shows a large map depicting the location and area of native American lands in the central and western United States. Three insets-arrayed in horizontal sequence and placed atop the larger map-show a smaller map of the United States, each depicting the area of native American land for the years 1819, 1894, and 2000. The math-centred question reads: "About what percentage of native American lands had the government taken over by 1894?" To solve, one must first "estimate (area)" in two maps, then 
compute "\% change" to assess the rapidly dwindling area of Native American land.

\subsection{Data File}

Data Files are call-outs that present a variety of numerical data and factual information (e.g., statistical tidbits), as part of a two-page-long scenario (say, of historical and/or cultural importance) that the reader must study in order to address the accompanying questions/tasks. There are five Data File call-out boxes in the textbook, four (or $80 \%$ ) of which sport one or more mathematical representations and involve mathematics work. In all, there are five mathematics representations appearing on these structures - three line graphs and two numerical tables. Also, all Data File call-outs pose two sets of questions-one always involves mathematics (albeit sometimes in simple forms) and the other is always social studies-centred.

To illustrate, consider a Data File in which six short items of factual information are provided regarding TV events of the 1970s. Below this fact list, a line graph depicts “Average Weekly Hours of TV Viewing” for three distinct population groups (children, teens, and adults 18 and over) for the period 1970-1998. In the two sets of questions that follow, students are first asked to do some historical analysis by answering: "In what ways did television change to reflect American society in the 1970s? What factors might have influenced these changes?" (p. 809). In the second math-centred question, students are instructed to consult the Internet for data on TV ownership and daily watching hours and then graph such data.

\subsection{Assessment Sections}

Following common textbook design practice, at the end of each chapter of The Americans there is an Assessment section that spans two facing pages. On one page there are chapter review questions/tasks, whereas on the other we find Texas Test Practice, which poses problems emulating standardized test questions (in this case, the State of Texas Assessments of Academic Readiness, STAAR). Of the 27 Chapter Assessment sections, eight (nearly 30\%) contain mathematics questions, and these questions appear exclusively in the Texas Test Practice portion of the section.

Because the Texas Test Practice portion is meant explicitly as a context for exercising (standardized) test-taking skills, mathematics questions are modelled strictly after the STAAR format (four-item multiple choice). Additionally, they are always anchored in one or two mathematical representations that depict some socially relevant data. In all, 14 mathematical representations appear across Assessment sections: eight line graphs, four bar graphs, and one numerical table.

A total of 10 mathematics questions are associated with the total eight test practice problems. Questions most frequently require students to work on "computing" values, "identifying trends", and "\% change". As an example, con- 
sider a problem in which two line graphs are presented that show U.S. oil consumption and production in the yearly period between 1965 and 1979. The oil production graph shows a nearly flat output over the 14-year period, whereas the oil consumption graph shows an increase of about $70 \%$ for the same period. In the associated multiple-choice questions, then, one is asked to "identify trends" in the graphs to explain how the oil crises of the 70's affected the U.S. economy.

\subsection{Other}

Lastly, but very significantly, mathematics is present across many other structures and sections of the book. First and most substantively, mathematics figures as the sole goal of two End-of-Chapter Skill Activity sections-titled "Interpreting Graphs and Charts" (p. 665 SK1) and "Creating Graphs and Charts" (p. 827 SK1), respectively. These are two-page "special" end-of-chapter sections and both are structured as tutorials that walk students through the steps of reading and analysing graphs, charts, and tables, and finally how to create graphs based on their own data collection. Both tutorial sections end with a proposed problem, again structured to walk students through analysing a given graph, then creating their own (e.g., how to label axes in graphs or rows/columns in tables). All in all, there is a hefty presence of mathematics in canonical instructional form.

Additionally, mathematics shows in two Geography Spotlight sections (a two-page, end-of-chapter section) and one Economic Spotlight section. As always, these sections sport several math representations and problems, all centred on exercising students' graphing abilities.

Finally, mathematics appears on additional seven occasions, always in the form of a representation with no questions/tasks attached. These representations are meant to illustrate the arguments of the main text, although the text itself does not refer to them.

\section{Analysis (2): Characterizing and Critically Assessing the Mathematics Content}

Drawing on the above analysis, we now characterize those mathematical contents along several dimensions and then evaluate the general mathematical work and reasoning they likely elicit.

\subsection{The General Character of the Mathematics in the Textbook}

As outlined in our conceptual framework, the nature of the mathematics content reflects both the qualitative forms that mathematics takes in the text and how such forms are framed by book structures, large and small. To pinpoint the nature of the mathematics found in the book, therefore, we look at the intersection o: 1) the specific material instantiations of math, 2) the book structures (e.g., call-out boxes, end-of-chapter sections, and so on) in which those appear, and 3) the mathematical work/reasoning those contents afford. In doing so, first we 
find that throughout the book the target conceptual domain is overwhelmingly Cartesian graphing, followed at a distance by basics of percentages, basic computation, and alternative ways of representing quantity (e.g., infographics). Additionally, such content is manifested in a variety of material forms, instructional genres, and functional contexts of mathematics use.

\subsubsection{Material Forms}

Materially, as we have seen at length, mathematics shows mostly as: 1) visual representations, including line and bar graphs, tables, pie charts, and infographics (Table 1); and 2) mathematical questions (i.e., queries of mathematical nature that appear in math-dedicated structures such as Skillbuilder Interpreting Graphs, or other structures such as Geography Skillbuilder). This is in general alignment with how mathematics is instantiated in mathematics textbooks of the same grade level.

Note that line graphs comprise a full half of all mathematical representations appearing in the book (Table 1), and some form of Cartesian graph comprises more than $62 \%$ of all mathematical representations. More than $58 \%$ of all line graphs appear within a Skillbuilder Interpreting Graphs structure, and were thus associated with some form of mathematics exercising.

\subsubsection{Instructional Genres}

Genres of mathematical instructional practice include problems, tutorials/lessons, and assessments. Among these, by far the most common is the problem, which combines one or more mathematical questions attached to one or more representational forms (e.g., graphs, tables, and maps) (Figure 1), and which are ubiquitous in structures like Skillbuilder Interpreting Graphs, Geography Skillbuilders, Assessment sections, and End-of-Chapter Skill Practice sections. In fact, mathematics problems are so widespread and dominant that they appear linked to essentially every disciplinary content in the book (even pop culture), as well as the book's core organizing page layout structures. Again, an emphasis on problems as central elements in mathematics instruction aligns with how mathematics practice is traditionally enacted in classrooms. We will say more about problems in the next subsection.

Table 1. Mathematical representations and their distribution over textbook structures (2015 edition).

\begin{tabular}{ccccccc}
\hline \multirow{2}{*}{$\begin{array}{c}\text { Mathematical } \\
\text { Representations }\end{array}$} & $\begin{array}{c}\text { Skillbuilder } \\
\text { Graphs }\end{array}$ & $\begin{array}{c}\text { Geography } \\
\text { Skillbuilder }\end{array}$ & $\begin{array}{c}\text { Data } \\
\text { File }\end{array}$ & Assessment & Other & Total \\
\hline Line Graph & 28 & - & 3 & 8 & 6 & $45(50 \%)$ \\
Bar Graph & 5 & 1 & - & 4 & 1 & $11(12.2 \%)$ \\
Pie Chart & 9 & 1 & - & - & 4 & $14(15.6 \%)$ \\
Numerical Table & 4 & 2 & 2 & 1 & 6 & $15(16.7 \%)$ \\
Infographic & 2 & - & - & - & 3 & $5(5.5 \%)$ \\
Total: & $48(53.3 \%)$ & $4(4.4 \%)$ & $5(5.6 \%)$ & $13(14.4 \%)$ & $20(22.3 \%)$ & 90 \\
\hline
\end{tabular}


Tutorials and Assessments are yet additional genres of mathematics instruction found in The Americans. As we have considered, in the former students are shown steps to be followed in the execution of a task (e.g., graphing data), then given a problem that rehearses those steps. In the latter, students are shown either problems meant to test the apprehension of recently read material (in the case of chapter assessment sections) or problems targeted at exercising mathematics test-taking skills (in the case of the Texas Test Practice portion of Assessments).

\subsubsection{Functional Contexts for Mathematics Practice}

Functional contexts reflect the different uses to which mathematics is put across the various page and chapter structures in the text. For the most part, given the predominant problem-centred form of mathematics content, a prominent functional context is exercising mathematics skills and concepts, as prototypically embodied in the dedicated structure of the Skillbuilder Interpreting Graphs.

Directly related, the standardized test practice portion of end-of-chapter Assessment sections is a functional context that extends mathematics problem practice/exercising into the realm of formal test practice. Here we see very directly the consequences of high-stakes, state-mandated assessments-and the importance of mathematics test scores for school promotion and accountability (Moses \& Nanna, 2007)—on the character and ubiquity of mathematics content in the book. We return to this point in the discussion.

Finally, mathematics is also used as a (modest) tool to work on issues in social studies. This is most commonly seen in the history-mathematics integrated questions appearing in Skillbuilder Interpreting Graphs, as well as geography-mathematics ones found in Geography Skillbuilders. Such questions, however, do not engage mathematics in ways that allow students to critically probe substantive problems in society and history, as we elaborate next.

\subsection{Critically Evaluating the Mathematics in the Textbook}

Problems/questions are the prime medium for "doing mathematics" in The Americans and thus it behooves us to understand the specifics of that genre. As outlined in our framework, here we take a critical lens that compares and contrasts the math problems in the textbook to the various roles that mathematical problem solving has served in the classroom (Schoenfeld, 1992, 2007). We pay particular attention to the reasoning fostered by such mathematical forms and their uses in social studies thinking.

As discussed by Schoenfeld (2007), problems and problem solving are often means to focused (instructional) ends, such as "justifying the teaching of math" (by making problems relevant to daily life concerns, however tenuously), motivating the introduction of "new subject topics," and "routine exercising" a procedure or mathematics technique (on the assumption that repeated practice leads to memorization and learning). Problems and problem solving are also used as forms of entertainment (i.e., "problems as recreation"), as when prob- 
lems of some complexity are used to illustrate that mathematics can be fun and that mastering it leads to such fun (Schoenfeld, 1992). Further, "problems as assessment" refer to those instances in which a problem functions as summative assessment of students' grasp of a chapter's material or lesson, whereas "problems as performance" seek to train students for very specific instructional performances, such as test-taking.

Beyond a means to focused ends, mathematics problems and problem solving have also been framed as a set of skills that are worthy of learning in their own right, as ends in and of themselves. In this view, such skills become an explicit target of instruction and a hierarchy of skills and problems is usually devised (e.g., routine and non-routine problems) that organize instruction of the subject. "Typically, problem solving techniques (such as drawing diagrams, looking for patterns when $n=1,2,3 \ldots) \ldots$ are taught as subject matter, with practice problems assigned so that the techniques can be mastered" (Schoenfeld, 1992: p. 338). As a result of instruction, then, students' mathematical toolset is supposed to contain such skills and techniques alongside facts and procedures.

Considering all problem instances catalogued and described in the prior analysis, it is clear that mathematics problem solving embodied in The Americans variously represent most (if not all) of these categories, and each problem almost always embodies various categories simultaneously. For example, most (but not all!) mathematics problems/questions attempt to "justify the teaching of math" by couching the problem context (to a greater or lesser extent) in a social studies theme/issue. At the same time, some of these same questions also function as "assessment" and "routine exercising" contexts in which students practice procedures of varying difficulties. This pattern is manifested across all page and chapter structures in which a mathematics question appears.

Similarly, a few problems may be said to fall into the category of "problems as recreation," such as when extended, open-ended tasks-notably those in Data Files call-outs-require students to gather and analyse data (say, by searching the Internet) and present conclusions to the class. Problems appearing in Assessment sections, on the other hand, are very narrowly framed as "problems as assessment" of recently covered material or, more commonly, as "problem as performance" context for students' exercising test-taking skills (as those in the Texas Test Practice portion of Chapter Assessment sections).

By and large, however, problems and tasks in The Americans frame mathematics and its problem solving practices as a set of skills to be acquired (see Glasthal, 1996 for other examples). There is abundant and unmistakable evidence for this inference. Perhaps most obviously, the very language of the $10^{\text {th }}$ grade Social Studies state standards (TEKS, printed in the opening pages of the book) positions mathematics squarely in that realm. To wit, all math-related TEKS appear under the rubric of Social Studies Skills and TEKS 29H explicitly states students should be able "to use appropriate skills to analyse and interpret social studies information such as maps, graphs, presentations, speeches, lectures, and political cartoons" (emphasis added). Unsurprisingly, the language of 
the standards carries over to the very core of The Americans, as seen in the structures Interpreting Graphs Skill builder, Geography Skill builder, and End-of-Chapter Skill Activities. Likewise, throughout the End-of-Chapter Skill Activities "Creating Graphs and Charts," the language of skill building is on display in the subsection's headings "Defining the skill" (of how to create a graph), "Understanding the skill," and "Applying the skill" (as in "now do it yourself").

Most glaringly, beyond the ubiquitous language of math-as-skill that dominates the text, the format of mathematical problems in the book unmistakably points to a skill-centred orientation to that discipline. To begin, note that mathematics questions are short-term and always single-answer. Furthermore, each question in a given problem targets a single skill and the skill required to solve the first question underlies that of the second question-a sort of sequence/hierarchy of skills. As in any skills-based take on mathematics problems, skills are well-defined and circumscribed-namely, "read value" (e.g., from y or $\mathrm{x}$ coordinate or numerical table), "assess slope," "find $\mathrm{min} / \mathrm{max}$," calculate "\% change," "identify trend," "estimate (area/distance)," "rate of progress," and others, as captured in our analytical categories (considered previously). Finally, skills embodied in problems/questions are practiced over and over, across book structures, disciplinary contents, instructional genres, and functional contexts.

Now mathematics educators would readily articulate several alternative ways to structure mathematics problems for conceptual development and skill building. To illustrate, let us consider a very different notion of mathematical problem solving-one with roots in the professional practice of mathematicians (Pólya, 1945). In this perspective, mathematics problem solving is a goal in its own right and it stands as the core process of mathematical inquiry. This process entails such "authentic" practices as generating and testing hypotheses, formulating conjectures, investigating patterns, devising alternative solutions, and seeking generalizations. Mathematical problem solving is thus a mode of inquiry that is exploratory and ever deepening, generative, and problem posing, rather than a circumscribed set of skills or techniques that are learned through repeated exposure and exercising (Schoenfeld, 1992; Schoenfeld, 2007).

To illustrate, we borrow Hollister's (1995) example of mathematical problem solving within social studies. Hollister posits that the social studies classroom should deploy mathematics in ways that empower students to carry out data-driven, critical historical and geographical inquiry. Referring specifically to social studies textbooks and what he sees as problematic historical narratives, Hollister (1995) suggests that students should be able to deploy mathematics as a means "to question the very generalizations and assumptions" (p. 14) found in such texts.

Hollister articulates his approach with an activity in which students investigate the changing population of the American bison, from the times Native Americans ruled the West to about 1990 . While the activity has wide ranging goals, a main target is to examine the (according to him) common textbook narrative that sustains the sharp decline in bison numbers from 1865 to 1875 
was due to "The Opening of the West"-that is, the coming of white settlers and their technologies (i.e., railroads, the rifle, barbed wires, and the steel plow) to the plains during that period. To advance his argument, Hollister leads students through analysing several bar graphs portraying bison population across various ranges of historical periods; each graph affords specific insights into trends in population numbers and raises specific questions about causal mechanisms (e.g., historical, environmental, and geographical) driving bison population change.

Starting by graphing the bison population from 1885 to 1983, Hollister shows both the decline in bison numbers and their posterior population growth and eventual saving from extinction. Next, by superimposing a graph with bison population data from 1865 to 1889 , students can better verify the sharp decline in numbers (from roughly 15,000 to about 1000) in the decade between 1865 and 1875 , as well as the even sharper recovery in modern times (1990). But by focusing on the population decline between 1865 and 1870, Hollister convincingly argues that bison numbers were already in significant decline before settler migration could have had a substantive effect on those animals. By building a simple spreadsheet model, Hollister then asks students to project bison numbers prior in time as well as into the far future, effectively engaging students in simultaneous mathematical and historical conjecturing. As they progress in their work, students come to ask: “...do the data support or refute the textbook interpretation of the American bison's decline? Can I make a different interpretation from the historians? How can the data both help us and hinder us in interpreting what happened 'historically'?" (Hollister, 1995: p. 14). And in the generative manner of Pólya's mathematical problem solving, Hollister states: "You will note that we have raised far more questions than we have given answers... Furthermore, these questions will lead us in a trail across disciplines" (p. 16), linking them in epistemologically deep ways.

Clearly, Hollister's take on mathematical problem solving substantively departs from that embodied in The Americans. Significantly, Hollister's activity engages the same mathematics skills as those targeted in the book and thus addresses the same state standards (TEKS). But it does so through an activity structure in which mathematics skills are evoked and practiced in the service of conceptual goals and powerful mathematical practices, such as hypothesizing with math, making predictions, and modelling. Within that context of problem solving, testing and practicing "skills" is integral to pursuing a systematic solution to questions that students continuously revisit or pose anew (Schoenfeld, 2007). Just as importantly, the goal of "breaking open" textbook assumptions and narrative, in and of itself, positions students in authoritative roles to pursue historical inquiry of critical and deep nature.

One may question the technical and practical feasibility of such an ambitious classroom and curricular approach. That, however, is not the substantive issue at stake. The question is that of student thinking and learning, in both social studies and mathematics, and the visions of literacy that animate decisions about curriculum and their instantiation in official textbooks. Unquestionably, there 
are different ways to implement standards-based instruction, as well as how those standards are represented in textbooks (Apple, 1992). The choices regarding the specific forms of implementing these standards, therefore, reflect specific ideological positions on the nature and goals of both mathematics and social studies literacy.

\section{Analysis (3): The "Density" and Shifting Patterns of Mathematics Content}

Based on the above, what is the relative "density" of mathematics content in the book? How has the "density" of mathematics content and its specific material manifestations changed over editions of The Americans textbook series and what trends might these shifts suggest? For clarity, we address each question separately.

\section{1. "Density" Matters}

To assess the relative presence of mathematical content in the book, we first summarize some basic numbers on the frequency of occurrence (Janks, 2010) of various structures and disciplinary content. To begin, at the page level and its constitutive call-out boxes, we find 31 Skillbuilder Interpreting Graphs, seven Science \& Technology, nine Economic Background, 33 Historical Spotlight, 55 Geography Skillbuilder, and 66 Key Player (which describes central historical figures). All in all, mathematics has a very prominent position when compared to Science \& Technology and rivals even some substantive social studies content (e.g., Historical Spotlight and Economic Background call-outs).

Considering page "real-estate" presence (Nichols, 2003), mathematics is materially manifested most dominantly as representations and questions/tasks. Starting with the former, in total there are 90 mathematical representations-or slightly more than three per chapter, on average (Table 1). So pictorially, too, mathematics has a dominating presence when compared to all visual representations (e.g., photographs and models) linked to Science and Technology, which totals 52. Perhaps most strikingly, the total 90 mathematical representations are comparable even to the total 96 maps (geographical and political) in the book.

As for proposed questions and tasks, there are 81 in all (three per chapter, on average) that centre on mathematics. In contrast, there are two problems in Science \& Technology in the whole book. And again, even when compared to Geography Skillbuilders' 110 geography problems (of which 13 are math-related), mathematics fares relatively well, given the social studies textbook context.

To be fair, treatment of Science \& Technology topics is most often embedded in the main narrative text (not in call-out boxes) and our count shows such topics take roughly the equivalent of 26 full pages. Additionally, the teacher edition of the 2015 text suggests another 13 distinct points along the textbook's narrative where connections to Science \& Technology (as a cross-cutting theme in history) could be made. 
Still, such numbers pale when compared with the overall "density" of mathematics disciplinary practice (broadly speaking), which stems not only from math's allocated real-estate and frequency of occurrence in the book, but also and fundamentally from its penetration across various disciplinary contents (history, economy, geography, politics), span (as in the variety of chapter and page structures that it inhabits), and multiple instructional genres in which it appears. In other words, mathematics (in some form) shows consistently throughout the text and it reaches across different types of call-out boxes, "special" sections, assessment sections, and others, and it requests active work from students (beyond reading). As an all-pervasive discipline, manifested in so many guises, practiced often and in different contexts, linked to most social sciences disciplines (economics, history, geography), mathematics establishes a significant "density" in the textbook. "Density" thus seems to act as a mechanism that reifies the studying of mathematics as a worthy pursuit within the curricular space of social studies.

\subsection{The Shifting Patterns of Mathematics Content in the Textbook Series}

How has the mathematics content changed over time and editions of the textbook? A look into the 1994 volume-a 969-page tome titled The Americans: $A$ History-reveals a very different landscape from what we have seen thus far. To the point, there are only 33 mathematical representations in that textbook, which is almost three times less than the 2015 edition. Usage of representational forms has also changed dramatically-the 1994 edition contains only seven line graphs, two bar graphs, no pie charts or infographics, and 24 numerical tables (22 of which depict data on election results!). Thus, tables were by far the dominant representational form ( $73 \%$ of the total) and there was only $1 / 8$ the number of graphs found in the latest (2015) edition.

Furthermore, the 1994 volume has 13 math-related questions in total, compared to 81 in the current edition-about $620 \%$ fewer questions. Significantly, none of these questions are linked to a mathematical representation; instead, all mathematics questions appear attached to political maps. The most common types of question are "compute (w/ map scale)" (four total) and "estimating (distance/area)" (four total). Tellingly, the canonical "rate of progress" problem already featured in one instance, a fact that illustrates some enduring features of school mathematics.

Also regarding the 1994 edition: it has no dedicated call-out structure for mathematics, such as the current Skillbuilder Interpreting Graphs; there is no standardized test practice section either; and there are no End-of-Chapter Skill Activities on analysing and creating graphs, as is currently the case. The absence of such dedicated structures and the myriad functional contexts for "doing math" that they now offer, of course, tell much about how mathematics has penetrated the textbook series. 
Moving to the 2002 volume-titled The Americans: Reconstruction to the $21^{\text {st }}$ Century, an edition immediately prior to our focal (2015) case-we find the graphical page layout of call-outs of the focal case has been adopted and mathematical representations and questions/tasks have dramatically increased in number (relative to the 1994 edition). Indeed, the 2002 and 2015 editions share more similarities than differences, including essentially the same call-out box types, chapter structures/sections, and overall content. Yet, differences are apparent that illuminate important trends in the use of mathematics in the social studies textbook series. Table 2 and Table 3 summarize key data.

Table 2. Comparing the mathematical content of the last two editions of The Americans. Significant percent changes highlighted.

\begin{tabular}{ccc}
\hline Textbook Structures and & \multicolumn{2}{c}{ The Americans' Textbook Editions } \\
\cline { 2 - 3 } Mathematical Forms & 2002 Edition & 2015 Edition \\
\hline Skillbuilder Interpreting Graphs & 31 & 31 \\
Representations & 49 & 48 \\
Questions & 48 & 47 \\
Geography Skillbuilder & 14 & 13 \\
Representations & 4 & 4 \\
Questions & 15 & 14 \\
Data File & 5 & 4 \\
Representations & 5 & 4 \\
Questions & 6 & 5 \\
Assessment Sections & 6 & $10(25 \% \uparrow)$ \\
Representations & 8 & $14(75 \% \uparrow)$ \\
Questions & 8 & $12(140 \% \uparrow)$ \\
Other & 5 & $20(122 \% \uparrow)$ \\
Representations & 79 & $6(100 \% \uparrow)$ \\
Questions & 3 & $68(10.3 \% \uparrow)$ \\
Total: & 91 & $80(18.2 \% \uparrow)$ \\
Representations & 76 & 81 \\
Questions & &
\end{tabular}

Table 3. Comparing the representational landscape across the latest two editions of The Americans. Significant percent changes highlighted.

\begin{tabular}{ccc}
\hline \multirow{2}{*}{ Mathematical Representations } & \multicolumn{2}{c}{ The Americans' ${ }^{\prime}$ Textbook Editions } \\
\cline { 2 - 3 } & 2002 Edition & 2015 Edition \\
\hline Line Graph & 36 & $45(25 \% \uparrow)$ \\
Bar Graph & 13 & $11(15.4 \% \downarrow)$ \\
Pie Chart & 13 & 14 \\
Numerical Table & 9 & $15(66.6 \% \uparrow)$ \\
Infographic & 5 & 5 \\
Total: & 76 & $90(\sim 16 \% \uparrow)$ \\
\hline
\end{tabular}


First, note that the total number of mathematics representations has increased by $18.2 \%$ in the latest edition (Table 2 ). Such gains are reflected most directly in the large increase (25\% growth) in the total number of line graphs and even larger $(66.6 \%)$ increase in numerical tables (Table 3 ). These observations point to two immediate tendencies: 1 ) the consolidating of mathematical representations (primarily graphs) as the premium medium for "doing mathematics" within the social science curriculum; and 2) the growing encroachment of such forms onto the total visual and real-estate space of the textbook. Cumulatively and over time, those work to naturalize the use of mathematics objects and practices within the social sciences classroom.

Second, notice that the total number of mathematical questions has remained almost constant (a small increase of less than 3\%) across the latest two editions (Table 2). But tellingly, from the 2002 to the 2015 edition, mathematics questions were stripped from certain parts of the book and added/moved to others. To wit:

- Standardized test practice problems, which appear in Texas Test Practice portion of Assessment sections (found on both editions), have seen an increase of $75 \%$ in overall numbers. Indeed, the number of Assessment sections that contain mathematics questions have themselves increased $33 \%$ overall. Together, these indicate a clear trend in increasing book space devoted to students' exercising standardized forms of mathematical problems-a purpose entirely extraneous to the social studies curriculum.

- Simultaneously, new instructional genres and functional contexts for "doing math" have been added to the latest edition that did not exist in prior editions. Most significantly, End-of-Chapter Skill Activity sections are new to the 2015 edition and the exclusive instructional mathematics focus of two such sections is indicative of the growing presence and influence of mathematics in the text's makeup. Perhaps most indicative of this encroachment trend, the content of those End-of-Chapter Skill Activity sections was already present in the 2002 volume, but placed in the Appendices at the end of the book-that is, beyond its substantive chapters and likely beyond students' and teachers' consideration. The not-so-subtle moving of such content into the core chapter structure of the book is strong evidence of the growing formalizing and institutionalizing of mathematics lessons within the disciplinary domains of the social studies textbook.

To summarize, the trajectory of mathematics usage and display across the 1994, 2002, and 2015 editions of The Americans clearly shows the turning of mathematics from a limited "tool" - used very sparingly and restricted to illustrating election results and some geographical issues-into a significant learning target of the book. The latest edition of the textbook clearly advances and further consolidates this trend by:

- Maintaining-indeed, slightly expanding-the total number of proposed mathematics questions (across all types of structures, Table 2). 
- Increasing the total number of mathematical representations by about $16 \%$ (Table 3).

- Increasing by $75 \%$ the space for the practice of standardized test-taking forms of mathematics problems.

- Increasing the penetration and span of mathematics, through the addition of new book structures (e.g., Economic Spotlight section) and instructional genres (End-of-Chapter Skill Activity) for doing mathematics.

Mathematics, therefore, seems to be exerting a colonizing force over the curricular space of the social studies, at least in its material manifestation in that area's textbook.

\section{Discussion}

As it appears in The Americans textbook series, over time mathematics has seen a dramatic increase in sheer presence in the book's core substance. This shift in "density" has been articulated with a concomitant and equally sharp shift in the purpose and uses to which that discipline is put in the social studies textbook. In turn, that has required yet another shift-namely, in the very content and kind of mathematics that is put forth in the text. Conceptually and materially, for instance, the mathematics content went from the use of numerical tables (and very few graphs) synthesizing historical and geographical facts/features to a focus (though not exclusive) on Cartesian graphing, explored through five different types of representational forms (and a now-dominant preference for line graphs and numerical tables). Significantly, whereas mathematics was previously an inert subject in the text, it is currently instantiated in various instructional genres-most prominently problems and questions, but also tutorial sections and others-that require some mathematical work, even if minimal. As evidence, recall that there are now 90 mathematics representations in the textbook. Of these, only seven appear without a mathematics question/task attached!

We believe that integrating mathematics into the social studies curriculum is welcome and positive. Indeed, as we have shown and in the spirit of making the most of existing textbook resources (Roberts, 2014a), it is not difficult to craft lessons that tap into the mathematics content of The Americans as means to advancing the democratic, civic, and participative notions of social studies education expressed in the state standards (e.g., students should "evaluate various means of achieving equality of political rights..." in TEKS 23B, and "evaluate the pros and cons of U.S. participation in international organizations and treaties," TEKS 19E) and widely advocated by educators (Barton \& Levstik, 2004).

That said, we find problematic the forms in which mathematics is currently instantiated in the book and the visions of mathematics literacy it espouses, explicitly and implicitly. Together, they work to impede the kinds of disciplinarily integrated approaches that can foster critical and principled reasoning across disciplines (Stevens et al., 2005). By the same token, the rendition of mathematics and mathematical problem solving as a set of skills severely limits how stu- 
dents might reason with and through mathematics, and thus how they might profitably learn the discipline, as illustrated by the bison population example.

To expand on those arguments, consider critical literacy approaches rooted in Freirian tradition (Freire, 1970) and their instantiation in mathematics education. Briefly, such approaches seek to empower students to develop a sense of themselves as social and historical actors and to engage the world in ways that are democratic and transformative. As Gutstein (2006) explains: "Students need to be prepared through their mathematics education to investigate and critique injustice, and to challenge, in words and actions, oppressive structures and acts—-that is, to "read and write the world" with mathematics" (p. 4). To read the world with mathematics entails using the discipline "to understand relations of power, resource inequities, and disparate opportunities between different social groups and to understand explicit discrimination based on race, class, gender, language and other differences" (Gutstein, 2006: pp. 25-26). Writing the world with mathematics translates into effectively using the discipline's body of knowledge and practices to effect change in students' lives, community, and society. Or, as Gutstein (2006) puts it, “... writing the world for youth [is] developing a sense of social agency” (p. 27).

To exemplify this approach, take an activity proposed by Bob Peterson (see Gutstein \& Peterson, 2006) in which mathematics is used to examine historical and social aspects of the Iraq war; all such questions related to financing the war efforts and how that might impinge on students' daily realities. To motivate the activity, Peterson begins with a quote from Dwight D. Eisenhower, the $34^{\text {th }}$ President of the United States: "Every gun that is made, every warship launched, every rocket fired, signifies in the final sense a theft from those who hunger and are not fed, those who are cold and are not clothed" (p. 40). Peterson also provides some baseline data that bear on proposed problems, such as the estimated cost of the war as of January 2013 (US\$ 810 billion).

On this background, the activity then poses a number of questions/tasks, such as: "4. A full scholarship to an out-of-state... major university... is worth about $\$ 41,000$ a year. Calculate how many school seniors could receive a fully paid four-year college education if the monies spent on the Iraq war-as of January 2013-had been set aside for college scholarships instead" (p. 41). Problem 5 reads: "A group called the National Priorities Project has estimated the amount of taxes that people in different states and cities have paid to fund the war in Iraq. Go to their website... and find your state or city. Using this number, calculate how many additional teachers and nurses could be hired in your community, assuming that each of them made the average national salary for their profession. In 2010-11 the national average teacher salary was $\$ 56,069$ and the national average nurse salary in 2011 was $\$ 67,800 ”$ (p. 41). In the end, Peterson seeks overall closure by asking students to revisit the opening Eisenhower quote ${ }^{2}$ in light of the contemporary political context.

${ }^{2}$ Incidentally, that Eisenhower quote is not included in The Americans. 
As with Hollister's (1995) activity on bison population analysis, Peterson's approach to mathematics problem solving in the context of social studies clearly departs from that embodied in The Americans. Rather than a skills-based orientation, both Peterson and Hollister position mathematics as a tool for purposeful social inquiry and critical investigation, not as subject matter and a goal of instruction in its own right. Indeed, in both cases, a core pedagogical goal is to empower students through the critical appropriation of the tools of mathematics and social studies, in ways that offer them an entry into the discursive practices of both. Strategically, within the specificities of their critical approaches, both Hollister and Peterson stay close to the state standards and intentionally expose students to contexts in which knowing mathematics skills, methods, facts, and strategies is indeed invaluable (e.g., the STAAR test). Hollister does so by emphasizing sophisticated modelling and data analytical methods, whereas Peterson takes a more "traditional" format of "search for information, sort it, and do various calculations."

Crucially, however, Hollister and Peterson differ in the particular meanings they attach to critical thinking and inquiry, and hence the choice of problem contexts and questions that students effectively investigate in each of those scenarios. To question textbook authority and narrative, as in Hollister's case, is certainly important and the data analytical means that he deploys effectively incorporate core disciplinary (mathematics and otherwise) practices of challenging theories, questioning interpretations, formulating and testing conjectures, and seeking alternative explanations and solutions. But it is a qualitatively distinct proposition to inquire about one's immediate reality-a reality often absent from textbook narratives (Sleeter \& Grant, 1991) - with the overt goal of questioning and (eventually) acting upon an unjust social order and structure. To borrow Gutstein's (2007) words, “...one can know abstractly that [the war costs billions of dollars], but it can become meaningful and real when one sees oneself and one's future in the numbers. Until then, it may remain just a large number with little connection to one's life" (p. 26). In Peterson's activity, therefore, a core learning goal is to raise students' critical consciousness and reasoned action through the critical and systematic mathematizing of social life.

The point to observe is that the different approaches to integrating mathematics in the social studies embodied in The Americans, Hollister's and Peterson's activity designs lead to students learning very different things; they are different pedagogies and epistemologies, and they target very different forms of reasoning and learning objectives. From the perspective of learning theories, a skills-based approach is most often associated with the decontextualized acquisition of "tools" that fail to support students' conceptual understanding (Schoenfeld, 2007; Greeno \& Hall, 1997). It is also epistemologically faulty insofar as it misrepresents the inquiry practices of mathematicians, social scientists, and historians (Stevens et al., 2005). Indeed, it communicates to students a narrow, limited, and limiting perspective on the uses of mathematics in the social 
studies context.

These observations are all the more relevant when we consider that a culture of mathematics as skill building, coupled with the use of skills-centred forms of problems/questions, is in strong alignment with an ideology of high-stakes testing and school accountability (Moses \& Nanna, 2007). Skill building underlies the culture of testing in its very format and nature-that is, in the focus on short-term problems, practice and repetition of well-bounded questions and solutions. The recent increase in Chapter Assessment sections in The Americans, and the even larger growth of Texas Test Practice within Assessment sections, undeniably point to that ideology taking a firm hold of the social studies textbook-a purpose we believe is predatory to the goals of social studies education and which works to naturalize the constant practice of mathematics across the curriculum.

The alternative, critical approaches we considered, on the other hand, operate under different ideological and epistemological commitments and utilize mathematical problem solving in social studies as a matter of practice-that is, as a routine, everyday way to inquire about the world through the use of all sorts of mathematical tools (skills included), in the pursuit of mathematics and social studies of substantive reach. But they themselves differ on the overall lessons they "impart" to students, the specific learning goals they address, and ultimately in their visions of the aims of literacy and education. While all "critical thinking and inquiry" is important, not all are the same and it is crucial to be explicit about the assumptions and aims underlying our instructional decisions.

\section{Final Words}

We have offered the case study of the mathematics content in The Americans social studies textbook as a first instance of this kind of analysis. Any such study can take a number of perspectives; we chose a critical stance to the problem as a way to highlight the powerful historical and mathematical thinking that students might engage in, given different ways of framing mathematical problems within the context of social studies. Regardless of our critical bent, however, our quantitative data show an unmistakable encroachment (first observed in 2002) of mathematics "material" in the social studies textbook and the attendant shift in its use, now instantiated as a specific focus of instruction and continuous practicing. Whether such patterns are widespread across textbooks and grade levels and what this means for the future of social studies education are some of the questions we hope to pursue if we are to realize our democratically shared visions for both social studies and mathematics education.

\section{Conflicts of Interest}

The authors declare no conflicts of interest regarding the publication of this paper. 


\section{References}

Apple, M. (2007). Education, Markets, and an Audit Culture. International Journal of Educational Policies, 1, 4-19.

Apple, M., \& Christian-Smith, L. (1991). The Politics of the Textbook. New York: Routledge.

Barton, K. C., \& Levstik, L. S. (2004). Teaching History for the Common Good. Mahwah, NJ: Lawrence Erlbaum Associates.

Coopmans, C., Vertesi, J., Lynch, M. E., \& Woolgar, S. (2014). Representation in Scientific Practice Revisited. Cambridge, MA: MIT Press. https://doi.org/10.7551/mitpress/9780262525381.001.0001

Freire, P. (1970). Pedagogy of the Oppressed. Continuum Press.

Glasthal, J. B. (1996). American History Math: 50 Problem-Solving Activities That Link Mathematics to Key Events in U.S. History. New York: Scholastic Inc.

Greeno, J. G., \& Hall, R. P. (1997). Practicing Representation: Learning with and about Representational Forms. Phi Delta Kappan, 78, 361-368.

Gutstein, E. (2006). Reading and Writing the World with Mathematics: Toward a Pedagogy for Social Justice. New York, NY: Routledge.

Gutstein, E. (2007). “And That's Just How It Starts": Teaching Mathematics and Developing Students' Agency. Teachers College Record, 109, 420-448.

Gutstein, E., \& Peterson, B. (2006). Rethinking Mathematics: Teaching Social Justice by the Numbers. Milwakee, WI: Rethinking Schools.

Hollister, B. C. (1995). Social Mathematics in the History Classroom. Social Education, 59, 14-16.

Janks, H. (2010). Literacy and Power. New York: Routledge.

Latour, B., \& Woolgar, S. (1986). Laboratory Life: The Construction of Scientific Facts. Princeton, NJ: Princeton University Press.

Lee, V. R. (2010). Adaptations and Continuities in the Use and Design of Visual Representations in US Middle School Science Textbooks. International Journal of Science Education, 32, 1099-1126. https://doi.org/10.1080/09500690903253916

Marino, M. P. (2011). High School World History Textbooks: An Analysis of Content Focus and Chronological Approaches. The History Teacher, 44, 421-446.

Moses, M. S., \& Nanna, M. J. (2007). The Testing Culture and the Persistence of High Stakes Testing Reforms. Education and Culture, 23, 55-72.

https://doi.org/10.1353/eac.2007.0010

Nichols, J. (2003). Methods in School Textbook Research. International Journal of Historical Learning, Teaching and Research, 3, 11-26.

Pickering, A. (1995). The Mangle of Practice.

Pólya, G. (1945). How to Solve It. Princeton, NJ: Princeton University Press.

Roberts, S. L. (2014a). Effectively Using Social Studies Textbooks in Historical Inquiry. Social Studies Research and Practice, 9, 119-128.

Roberts, S. L. (2014b). A Review of Social Studies Textbook Content Analysis since 2002. Social Studies Research and Practice, 9, 51-65.

Schoenfeld, A. (1992). Learning to Think Mathematically: Problem Solving, Meta-Cognition, and Sense Making in Mathematics. In D. Grouws (Ed.), Handbook for Research on Mathematics Teaching and Learning (pp. 334-370). New York: McMillan. 
Schoenfeld, A. (2007). Problem Solving in the United States, 1970-2008: Research and Theory, Practice and Politics. The International Journal of Mathematics Education, 39, 537-551. https://doi.org/10.1007/s11858-007-0038-Z

Sleeter, C. E., \& Grant, C. A. (1991). Race, Class, Gender, and Disability in Current Textbooks. In M. Apple \& L. K. Christian-Smith (Eds.), The Politics of the Textbook (pp. 78-110). New York, NY: Routledge.

Stanic, G., \& Kilpatrick, J. (1989). Historical Perspectives on Problem Solving in the Mathematics Curriculum. In R. Charles \& E. Silver (Eds.), The Teaching and Assessing of Mathematical Problem Solving (pp. 1-22). Reston, VA: National Council of Teachers of Mathematics.

Stevens, R., Wineburg, S., Herrenkohl, L., \& Bell, P. (2005). The Comparative Understanding of School Subjects: Past, Present, and Future. Review of Educational Research, 75, 125-157.

Wade, R. C. (1993). Content Analysis of Social Studies Textbooks: A Review of Ten Years of Research. Theory and Research in Social Education, 21, 232-256.

Ward, K. (2006). History in the Making: An Absorbing Look into How American History Has Changed in the Telling over the Last 200 Years. New York: The New Press. 